\title{
Influence of ion implantation on the electroabsorption spectrum in photorefractive GaAs/AlGaAs quantum wells
}

\author{
E. Miśkiewicz, ${ }^{*}$ M. Wichtowski, E. Weinert-Rączka \\ Faculty of Electrical Engineering, West Pomeranian University of Technology, al Piastów 17, 70-310 Szczecin
}

Received February 18, 2016; accepted March 29, 2016; published March 31, 2016

\begin{abstract}
Dark resistivity and electrooptic effect are two of the necessary conditions crucial for the photorefractive effect to occur. Proton implantation used for increasing the dark resistivity of semiconductor heterostructures can influence the electrooptic coefficient. In this paper, there are presented the results of absorption and electroabsorption spectra measurements in semi-insulating $\mathrm{GaAs} / \mathrm{Al}_{0.3} \mathrm{Ga}_{0.7} \mathrm{As}$ multiple quantum well (MQW) structures. The dependence was analyzed between the electroabsorption amplitude near the excitonic peaks and the different proton implantation parameters.
\end{abstract}

Semi-insulating multiple quantum wells (SIMQW) comprise a special class of photorefractive materials as they have very high sensitivity and short response time. The highest photorefractive response is observed for the photon energies close to exciton transitions [1-2]. Due to the strong absorption near the excitonic resonance, SIMQW samples are usually designed as thin films.

Due to their unique properties, photorefractive multiple quantum well structures can find a broad range of applications from dynamic holography and optical signal processing to generation and transmission of solitons [3-7]. Low temperature grown MQWs can be used in ultra-fast communication systems as detectors and emitters [2].

The semiconductor MQW structure needs to possess certain material properties to be photorefractive. One of the requirements is relatively high dark resistivity. Conventionally MBE (molecular beam epitaxy) or MOCVD (metal-organic chemical vapor deposition) grown structures do not fulfill this requirement. They always possess a certain amount of impurities acting as shallow dopants and increasing the conductivity of the material.

One of the most common techniques to ensure semiinsulating properties is proton implantation which creates the traps compensating residual shallow dopants. It is not the only one available type of ion implantation but it presents several advantages over the other types. For example, protons as the lightest ions have the biggest possible penetration depth and can be used on relatively thick structures.

Another method is epitaxial growth at low substrate temperatures. Such LTG (low temperature growth)

*E-mail: eliza.miskiewicz@zut.edu.pl photorefractive MQWs have some interesting properties such as ultra-short carrier trapping time [2].

One of the most popular multiple quantum well systems is the GaAs/AlGaAs MQW structure operating in Franz-Keldysh geometry, with an external electric field applied along the layers of quantum wells [1-2],[8-9]. In this setup the high intensity electric field ionises the excitons and shortens their lifetime. This phenomenon manifests itself in the absorption spectrum as broadening and decreasing of peaks corresponding to excitonic transitions and is thus called electroabsorption [8].

The main aim of the present study was to examine the influence of proton implantation (used for increasing the dark resistivity) on the absorption and electroabsorption spectra of GaAs/AlGaAs multiple quantum well structures. Information about the relation between the implantation doses and electro-optical properties of a material would be helpful in a variety of design tasks including photorefractive devices and planar photorefractive waveguides [2-7].

Examined structures were $\mathrm{GaAs} / \mathrm{Al}_{0.3} \mathrm{Ga}_{0.7} \mathrm{As}$ multiple quantum wells manufactured at the Institute of Electronic Materials Technology (ITME) in Warsaw using a metalorganic chemical vapour deposition method (MOCVD). The samples included 100-period superlattices consisting of $7 \mathrm{~nm} \mathrm{GaAs}$ wells and $6 \mathrm{~nm}$ AlGaAs barriers.

Table 1. Values of implantation doses for the studied series of samples. The doses were the same for each implantation energy $(80 \mathrm{keV}$ and $160 \mathrm{keV})$. Sample series A was not implanted.

\begin{tabular}{|c|c|c|c|c|}
\hline & $\begin{array}{c}\text { Series } \\
\text { A }\end{array}$ & $\begin{array}{c}\text { Series } \\
\text { B }\end{array}$ & $\begin{array}{c}\text { Series } \\
\text { C }\end{array}$ & $\begin{array}{c}\text { Series } \\
\text { D }\end{array}$ \\
\hline $\begin{array}{c}\text { Doses } \\
{\left[\times 10^{12} \mathrm{~cm}^{-2}\right]}\end{array}$ & 0 & 1 & 1.5 & 2 \\
\hline
\end{tabular}

The structure was divided into four parts. One part was not irradiated. The other three were proton implanted at two energies, $160 \mathrm{keV}$ and $80 \mathrm{keV}$, with the same proton doses for each energy and then divided into a few samples. The specific values of implantation fluxes are given in Table 1. To perform electro-optical measurements, two samples from each of the series were chosen. The selection was based on the current-voltage 
characteristics, most representative for a given sample series.

The experiment was conducted in the wavelength range of the excitonic resonance $(810 \mathrm{~nm}-850 \mathrm{~nm})$, and the samples were operating in the Franz-Keldysh geometry.

The scheme of the setup used for absorption measurements is presented in Fig. 1. The studied sample was illuminated by a light beam from a tunable $\mathrm{Ti}: \mathrm{Al}_{2} \mathrm{O}_{3}$ laser. A cylindrical lens was used to form the beam crosssection to an ellipse, to illuminate the area with a possibly uniform electric field. For convenience, the light beam was split into a reference beam with intensity $I_{0}$, (measured by the photodiode PD1) and a beam illuminating the MQW structure, with intensity $I_{0}$. After passing through the sample, the beam intensity $I_{T}$ was measured by the photodiode PD2.

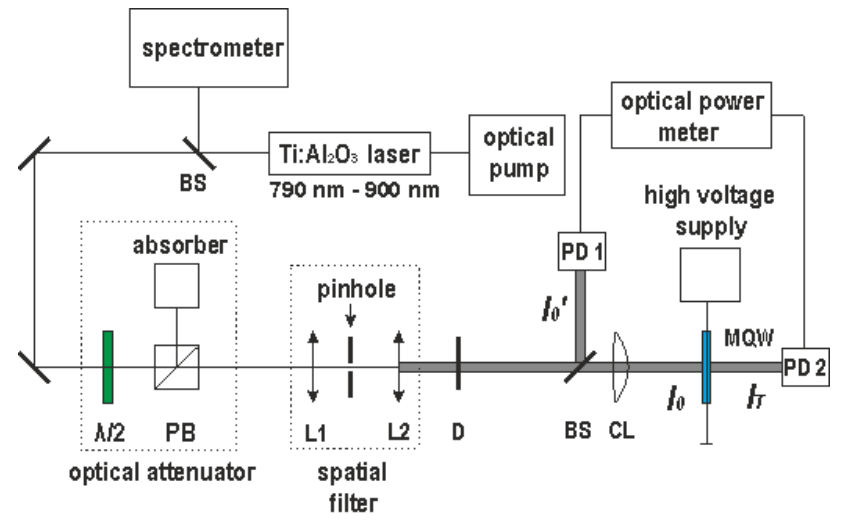

Fig. 1. Experimental setup for the measurements of absorption and electroabsorption spectra. Used abbreviations: BS - beamsplitter, $\lambda / 2$ - half-wave plate, $\mathrm{PB}$ - polarising beamsplitter, D - diaphragm,

L1, L2 - focusing lenses, CL - cylindrical lens, PD1, PD2 - Si photodetectors (photodiodes).

The absorption coefficient $\alpha$ was determined by measuring the $I_{T}$ to $I_{0}$ ' ratio, marked as $T^{\prime}$. The intensity $I_{T}$ is given by the relation $I_{T}=I_{0}(1-R)^{2} \exp (-\alpha L)$, where $R$ is the reflectance of the sample facets. The intensity $I_{0}^{\prime}$ can be noted as $I_{0}^{\prime}=I_{0} h(\lambda)$, where $h(\lambda)$ is the splitting ratio of a beamsplitter (the splitting ratio also includes losses introduced by the cylindrical lens). The measured $T^{\prime}$ is then given by the following expression:

$$
T^{\prime}=\frac{I_{T}}{I_{0^{\prime}}}=\frac{I_{0}(1-\mathrm{R})^{2} \exp (-\alpha L)}{I_{0} h(\lambda)}=\frac{\exp (-\alpha L)}{h(\lambda)}(1-R)^{2}
$$

where $L$ is the sample thickness (approximately $1.3 \mu \mathrm{m}$ ). The multiple reflections inside the sample were neglected due to strong absorption. From Eq. (1) was derived the absorption coefficient $\alpha$ :

$$
\alpha=\frac{1}{L} \ln \left(\frac{1}{T^{\prime}}\right)+\frac{1}{L} \ln \left(\frac{1}{h(\lambda)}\right)+\frac{1}{L} \ln \left((1-R)^{2}\right)
$$

After obtaining absorption coefficients measured with and without external electric field, $\alpha(\lambda, E)$ and $\alpha(\lambda, 0)$, respectively, the change of the absorption coefficient $\Delta \alpha(\lambda)$ can be calculated using the relation:

$$
\Delta \alpha(\lambda)=\alpha(\lambda, E)-\alpha(\lambda, 0)
$$

It was measured that reflectance $R$ changes with the light wavelength but is practically insensitive to the electric field, as shown in Fig. 2. Therefore neglecting the reflected light affects the accuracy of calculated absorption spectra (error increases above 10\%), but it has a relatively small impact on electroabsorption, as the R-dependent elements are cancelled.

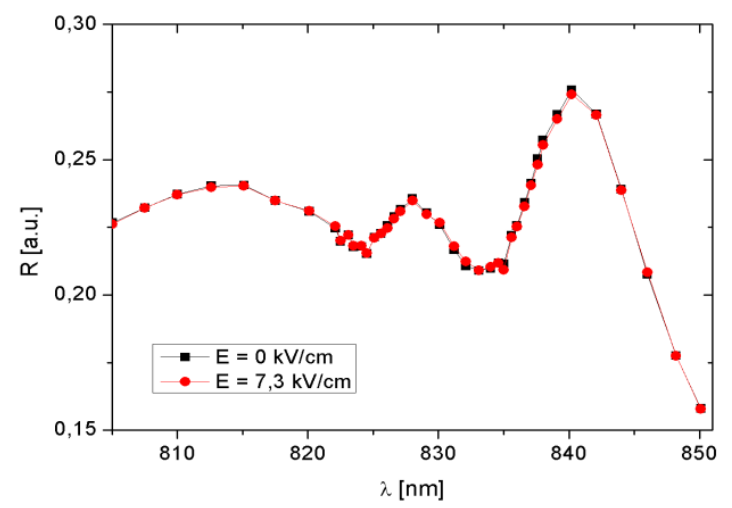

Fig. 2. Example of measured reflectance $R$ as a function of the light wavelength for sample B1.

Measurement results are shown in Figs. 3-5 and in Table 2 .

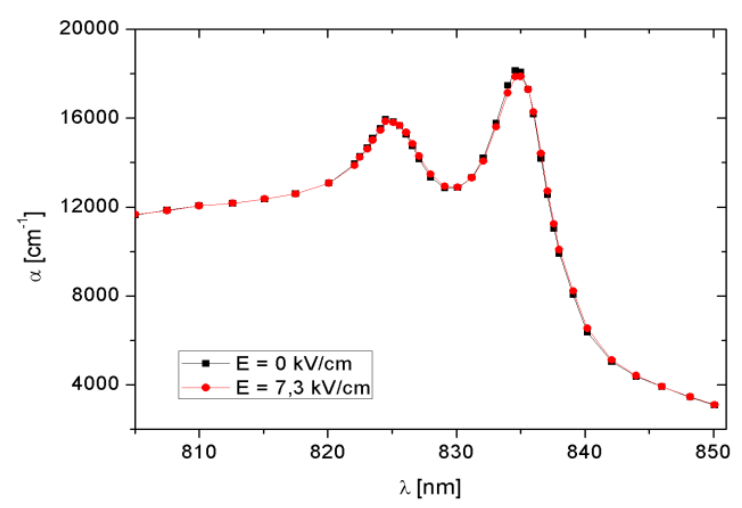

Fig. 3. Example of the measured absorption spectrum obtained for sample B1; black squares and red dots represent absorption without applied electric field and with applied electric field $E=7.3 \mathrm{kV} / \mathrm{cm}$ respectively; solid lines are linear point-to-point joints. Symbols hh and lh designate heavy hole and light hole resonance peaks, respectively.

Figure 3 presents an example of the absorption spectrum obtained for the sample with the smallest implantation dose (B series). The lines joining experimental data were added to guide the eye. 


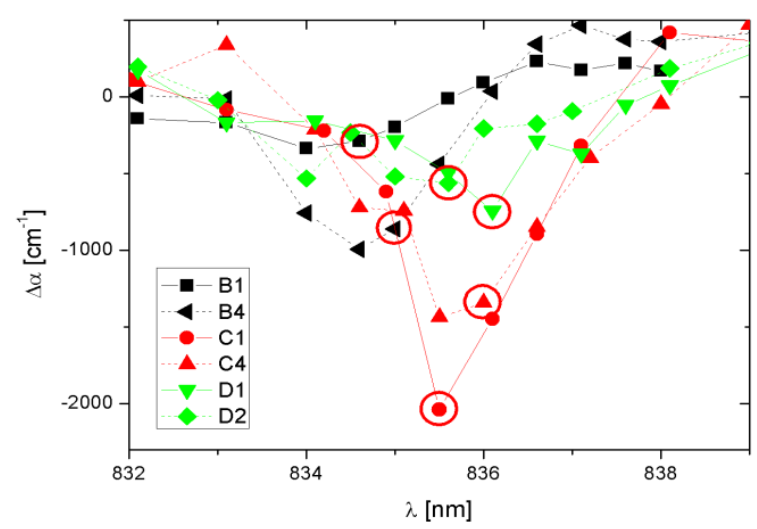

Fig. 4. Measured changes of absorption in the vicinity of a heavy hole peak for each examined sample. The red rings mark the points with the highest absorption without applied electric field.

Table 2. Values of changes in the absorption coefficient at heavy and light hole peaks for the samples of different implantation doses.

\begin{tabular}{|c|c|c|c|c|}
\hline \multicolumn{5}{|c|}{ Values of the changes in the absorption coefficient } \\
\hline & \multicolumn{2}{|c|}{ at light hole peak } & \multicolumn{2}{|c|}{ at heavy hole peak } \\
\hline & $\begin{array}{c}\lambda \\
{[\mathrm{nm}]}\end{array}$ & $\begin{array}{c}|\Delta \alpha| \\
{\left[\mathrm{cm}^{-1}\right]}\end{array}$ & $\lambda[\mathrm{nm}]$ & $\begin{array}{c}|\Delta \alpha| \\
{\left[\mathrm{cm}^{-1}\right]}\end{array}$ \\
\hline Sample B1 & 825.1 & 32.674 & 834.6 & 287.621 \\
\hline Sample B4 & 825.6 & 355.490 & 835.0 & 859.495 \\
\hline Sample C1 & 826.1 & 261.435 & 835.5 & 2038.079 \\
\hline Sample C4 & 826.6 & 440.033 & 836.0 & 1342.483 \\
\hline Sample D1 & 826.6 & 154.602 & 836.1 & 743.376 \\
\hline Sample D2 & 826.1 & 641.675 & 835.6 & 562.372 \\
\hline
\end{tabular}

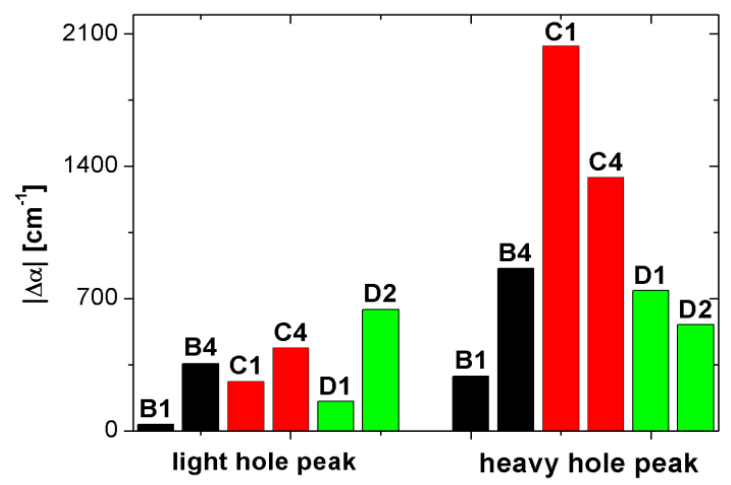

Fig. 5. Visualization of absorption changes for each measured sample, based on the data included in Table 2 .

Figure 4 shows absorption coefficient changes $\Delta \alpha(\lambda)$ calculated for the wavelengths near the heavy hole excitonic resonance. The red rings mark the points representing $\Delta \alpha$ at the heavy hole peaks. Some of those values vary from the highest obtained $\Delta \alpha$ for a given sample. The differences are relatively small and are in the range of the measurement uncertainty.

Table 2 contains the values of absorption changes $\Delta \alpha$ at the light hole and heavy hole peaks along with corresponding wavelengths. Figure 5 represents those values in the form of a bar graph. The differences between the peak central wavelengths are in the range of $0.5 \mathrm{~nm}$ within the sample series. Those changes could be due to equipment and measurement inaccuracy.

A significant difference in $\Delta \alpha$ between the samples with the same implantation doses was observed. This can be caused by a number of factors. One of them is measurement inaccuracy. The second one could be the fact that the electric field $E$ within the sample is calculated assuming the relation $E=U / l$ (where $U$ is the DC voltage applied to the sample and $l$ is the electrodes spacing). The electrodes were hand-made and each sample has a different distance between the electrodes and slightly irregular electrode edges. Any inaccuracy in the measurement of $l$ will result from a different intensity of the electric field applied to a given sample. Another factor that may influence the measurements are imperfections of the substrate etching process, which could change the samples thickness $L$ and influence the reflection conditions during the experiment.

Taking into account the influence of the issues discussed in the last section and the limited number of measured samples, it is difficult to find the relation between implantation doses and electroabsorption magnitude. However, the results show that any of the applied doses of proton irradiation did not decrease electroabsorption significantly. Furthermore, samples from the $\mathrm{C}$ series seem to exhibit the highest absorption change at the heavy hole peak. The $\Delta \alpha$ values obtained in the measurements are consistent with the results presented in study [10] for an analogical structure with similar implantation.

\section{References}

[1] P. Yeh, Introduction to photorefractive nonlinear optics (New York, Wiley \& Sons 1993).

[2] D.D. Nolte, Photorefractive effects and Materials (New York, Springer Science+Bussines Media 1995).

[3] M. Carrascosa, F. Agulló-Rueda, F. Agulló-Lopez, Appl. Phys. A 55, 25 (1992).

[4] D.D. Nolte, Opt. Lett. 19(11), 819 (1994).

[5] P. LiKamWa, A.M. Kan'an, IEEE J. Selected Topics in Quantum Electronics 2(3), 655 (1996).

[6] A. Ziółkowski, J. Opt. 14(3), 035202 (2012).

[7] A. Ziółkowski, Opt. Express 22( 4), 4599 (2014).

[8] Q. Wang, R.M. Brubaker, D.D. Nolte, M.R. Melloch, J. Opt. Doc. Am. B 91626 (1992).

[9] Q. Wang, D.D. Nolte, M.R. Melloch, Appl. Phys. Lett. 59256 (1991).

[10] S. Balasubramanian, I. Lahiri, Y. Ding, M.R. Melloch, D.D. Nolte, Appl. Phys. B 68, 863 (1999). 